\title{
A Study of Lipid Profile in Type 2 Diabetic Punjabi Population
}

\author{
Singh $^{1}$, G. and Kumar ${ }^{2}$, A.K. \\ ${ }^{1}$ Research Scholar, Department of Sports Science, Punjabi University Patiala (India) Email: \\ drgurdeep_sahni@yahoo.co.in \\ ${ }^{2}$ Assistant Professor, Department of Sports Science, Punjabi University Patiala (India) Email: \\ akashokin@gmail.com
}

\section{Abstract}

Aims: The purpose of the study was to observe the lipid profile of type 2 diabetics in the male Punjabi population. $\operatorname{Method}(s)$ : A total of 120 Type 2 diabetic men with an age range from 30 to 70 years volunteered to participate in this study. The fasting blood sugar (FBS) \& lipid profiles were recorded with standard procedure. Results: The mean age and FBS were $50.3 \pm 11.8$ years and $135.1 \pm 27.4 \mathrm{mg} / \mathrm{dl}$ respectively. There were $59 \%$ subjects with high total cholesterol (TC) levels and $98 \%$ were having increased LDL levels. $89 \%$ of the subjects were found with lower HDL level. Conclusion: It is concluded from the results of the present study that in type 2 diabetics dyslipidaemia was very common especially raised LDL levels. Results strongly suggest that further investigations should relate the effects of dyslipidaemia and abnormalities of insulin resistance in type 2 diabetics. And ethnic specific patterns of lipid profile in type 2 diabetics regardless of their glucose levels, suggests that ethnic-specific strategies and guidelines on risk assessment and prevention of CVD due to dyslipidemia are required.

Keywords: CVD, FBS, Dyslipidaemia.

\section{Introduction}

Dyslipidemia is one of the major cardiovascular disease (CVD) risk factors and plays an important role in the progress of atherosclerosis, the underlying pathology of CVD. The prevalence of dyslipidemia in type 2 diabetes is double with respect to the general population (Haffner, 1998). These are more complex abnormalities that are caused by the interrelation among obesity, insulin resistance and hyperinsulinism (Burstein et al., 1970 \& American Diabetes Association, 1998). According to Freedman et al (1999), when the overweight subjects were compared with their respective thinner counterparts, they presented 2.4 to 7.1 times higher probability to have an elevated total cholesterol, LDL cholesterol, triglycerides and blood pressure as well as 12.6 times higher probability to have hyperinsulinemia. It is worth to emphasize that the fatty tissue is exclusively related to risk factors, such as the altered insulin and lipid profile, which can contribute to the development of the insulin resistance syndrome, which comprises several risk factors for the emergence of cardiovascular complications (Gower, 1999). In patients with type 2 diabetes, which is equivalent to CHD (Juutilainen et al, 2005), it is most commonly characterized by elevated TG and reduced HDL-C (Goldberg 2001). These abnormalities can be present alone or in combination with other metabolic disorders. The prevalence of dyslipidaemia varies depending on the 
population studied, geographic location, socioeconomic development and the definition used (Wood et al, 1972; Berrios et al, 1997). Very few cross-sectional studies have evaluated the relationship between lipid and blood glucose concentrations in type 2 diabetics in Punjabi population. The present study was planned to identify the prevalence of abnormalities in lipid profile among type 2 diabetic Punjabi population.

\section{Materials and Methods}

One hundred and twenty type 2 diabetic male patients belonging to Patiala district of Punjab were selected as subjects after obtaining their informed written consent and their age ranged from 30-70 years. The objectives of the present study were thoroughly explained to them. Clinical history was also documented and following exclusion criteria were usednot taken any steroid therapy in past 3 months, any liver, kidney or cardiac failure, neoplasm and patients who were on any type of anti-lipidemic therapy. The study protocol was reviewed and approved by the Ethics Committee of Punjabi University, Patiala. The serum was separated immediately after obtaining the blood sample (overnight fasting) by using centrifugation for 10 minutes. Fasting blood glucose concentration and Lipid Profile [Total Cholesterol (TC), HDL, VLDL \& Triglycerides (TG)] were measured using Blood Analyzer. The appropriate chemical testing kits were used. LDL was calculated by using Friedewald formula: $\mathrm{LDL}=\mathrm{TC}-(\mathrm{TG} / 5)$ HDL. For the descriptive and inferential statistics SPSS version 16 was used.

\section{Results \& Discussion}

Table 1 shows the mean values of age and fasting blood sugar were observed to be $50.3 \pm 11.8$ years and $135.1 \pm 27.4 \mathrm{mg} / \mathrm{dl}$ respectively. Results of the BMI in the present study indicate that our subjects were not obese but their mean fasting blood sugar level was more than the normal value.

Table 1. Mean \pm SD of Age $\&$ FBS of Type 2 diabetics

\begin{tabular}{lc}
\hline Variables & Mean \pm SD \\
\hline Age $($ years $)$ & $50.3 \pm 11.8$ \\
FBS $(\mathrm{mg} /$ dl) & $135.1 \pm 27.4$ \\
\hline
\end{tabular}

Table 2 shows the quantitative analysis of lipid profile of Type 2 diabetics and found that the mean total cholesterol $(203.9 \quad \pm 15.8 \quad \mathrm{mg} / \mathrm{dl})$, triglycerides $(151.1 \pm 17.7 \mathrm{mg} / \mathrm{dl})$, HDL $(47.7 \pm 6.2 \mathrm{mg} / \mathrm{dl}), \mathrm{LDL}(124.4 \pm 11.9$ $\mathrm{mg} / \mathrm{dl}), \operatorname{VLDL}(32.3 \pm 7.1 \mathrm{mg} / \mathrm{dl}) \& \mathrm{LDL}$ / HDL ratio $(2.63 \pm .37)$.

Table 2. Mean \pm SD of lipid profile of Type 2 diabetics Variables

$\begin{array}{lc}\text { Total Cholesterol,TC (mg/dl) } & 203.9 \pm 15.8 \\ \text { Triglycerides, TAG (mg/dl) } & 151.1 \pm 17.7 \\ \text { HDL-C (mg/dl) } & 47.7 \pm 6.2 \\ \text { LDL-C (mg/dl) } & 124.4 \pm 11.9 \\ \text { VLDL (mg/dl) } & 32.3 \pm 7.1 \\ \text { Ratio of LDL / HDL } & 2.63 \pm 0.37\end{array}$

TC- total cholesterol, HDL- high density lipoproteins, LDL-low density lipoproteins, VLDL- very low density lipoproteins, TG- triglycerides

The present study also analyzed the prevalence rate of hypercholesterolemia, hypertriglyceridemia, low HDL and high low density lipoproteinaemia among type 2 diabetics. It was found that 59\% Type 2 diabetics in this study had hypercholesterolemia, 53\% Hypertriglyceridemia and $98 \%$ abnormal LDL levels. In $89 \%$ of Type 2 diabetics, the HDL was less than $40 \mathrm{mg} / \mathrm{dl}$. Thus, the results of the present study shows that in Type 2 diabetics the dyslipidaemia is the most common abnormality and it was found in the level of LDL-C value.

\section{Discussion}


For the interpretation of serum lipid reference values, the guidelines of National Cholesterol Education Programme (NCEP) Adult Treatment Panel III (ATP III) were followed. According to NCEP-ATPIII guidelines, hypercholesterolemia is defined as TC > $200 \mathrm{mg} / \mathrm{dl}$, high LDL-C when value > 100 $\mathrm{mg} / \mathrm{dl}$, hypertriglyceridemia as TAG > $150 \mathrm{mg} / \mathrm{dl}$ and low HDL-C when value is $<40 \mathrm{mg} / \mathrm{dl}$. Dyslipidemia was defined by presence of one or more than one abnormal serum lipid concentration (Menik et al., 2005).

The results of the present study were in agreement with the previous research reports that dyslipidemia, overweight and obesity is a common association with type 2 diabetic patients (Haffner, 1998 and American Diabetes Association, 1998). In the present study $59 \%$ of the subjects had hypercholesterolemia and 98\% abnormal LDL levels. Type of dyslipidaemia reported among diabetic population is numerous in different places in world indicating that dyslipidemia can be influenced by the interaction of genetic and environmental factors (Carlos et al, 2001). The prevalence of the lipid abnormalities reported by Mexican nationwide survey done by Carlos et al, (2001) is similar to that observed in Turkish (Mahley et al, 1995) and other Asian populations, including Bangladeshi and Pakistani populations (Bhopal et al, 1999). Their study shows that $53 \%$ of the diabetic population had Hypertriglyceridemia. The present study is in agreement with the above report in relation to prevalence rates of hypertriglyceridemia among type 2 diabetic Punjabi population $(53 \%$ in the present investigation and 54\% in Mexican nationwide survey). It was also found that
98\% of Type 2 diabetics had high LDL levels and 59\% showed hypercholesterolemia. This observation further confirms that patients with Type 2 diabetes had co-incidence of several abnormal lipid profiles. It further confirms that dyslipidemia could have impact on the development of insulin resistance in type 2 diabetes mellitus. Menik et al (2005) reported a significant genetic association between development of insulin resistance and dyslipidemia among type 2 diabetic patients.

\section{Conclusion}

It is concluded from the results of the present study that type 2 diabetics were either overweight or type I obese and dyslipidaemia was very common. Results strongly suggest that further investigations should relate the effects of dyslipidaemia and abnormalities of insulin resistance in type 2 diabetics. And ethnic specific patterns of lipid profile in type 2 diabetics regardless of their glucose levels, suggesting that ethnicspecific strategies and guidelines on risk assessment and prevention of CVD due to dyslipidemia are required.

\section{References}

American Diabetes Association - Position Statement. Management of Dyslipidemia in Adults with Diabetes. Diabetes Care 1998, 21: $179-82$.

American Diabetes Association - Position Statement. Management of Dyslipidemia in Adults with Diabetes. Diabetes Care 2001, 24: Supp 1.

Bhopal, R., Unwin, N., White, M., Yallop, J., Walker, L., Alberti, K.G., Harland, J., Patel, S., Ahmad, N., Turner, C., Watson, B., Kaur, D., Kulkarni, A., Laker, M., Tavridou, A. 1999. Heterogeneity of coronary heart 
disease risk factors in Indian, Pakistani, Bangladeshi and European origin populations: cross sectional study. Brit. Med. J., 319: 215-20.

Burestin, M, Selvenick, H.R., Morfin, R. 1970. Rapid method for the isolation of lipoproteins from human serum by precipitation with polyanions. Lipid Res., 11: 583-583

Friedewald, W.T., Levy, R., Fredrickson, D.S. 1972. Estimation of the concentration of lowdensity lipoprotein cholesterol in plasma without use of the preparative ultracentrifuge. Clin. Chem., 18: 499-502.

Berrios, X., Koponen, T., Huiguang, T., Khaltaev, N., Puska, P., Nissinen, A. 1997. Distributionand prevalence of major risk factors of noncommunicable diseases in selected countries: the WHO Inter-Health Programme. Bull. World Health Organ. 75: 99-108.

Carlos, A., Aguilar, S., Gustavo, O., et al. 2001. High prevalence of low HDL cholesterol concentrations and mixed hyperlipidemia in a Mexican nationwide survey. J. Lipid Res., 42: 1298-307.

Freedman, D.S., Dietz, W.H., Srinivasan, S.R., Berenson, G.S. 1999. The relation of overweight to cardiovascular risk factors among children and adolescents: the Bogalusa Heart Study. Pediatrics, 103: 117582.

Gower, B.A. 1999. Syndrome X in children: influence of ethnicity and visceral fat. Am. $J$ Hum. Biol., 11: 249-57.
Goldberg, I.J. 2001. Clinical review 124: Diabetic dyslipidemia: causes and consequences. $J$. Clin. End. Metab., 86: 965-971.

Haffner, S.M. 1998. Management of dyslipidemia in adults with diabetes. Diabetes Care, 21: 160-78.

Juutilainen, A., Lehto, S., Ronnemaa, T., Pyorala, K., Laakso, M. 2005. Type 2 diabetes as a "coronary heart disease equivalent": an 18year prospective population-based study in Finnish subjects. Diabetes Care 28: 29012907.

Mahley, R.W., Erhan-Palaoglu, K., Atak, Z., et al. 1995. Turkish heart study: lipids, lipoproteins and apolipoproteins. J. Lipid Res., 36: 83959.

Menik, H.L., Sammanthi, J.S., Priyantha, W.T., et al. Significant genetic association between insulin resistance and total cholesterol in type 2 diabetes mellitus; Preliminary study.

NCEP: 2001. Summary of The Third Report of The National Cholesterol Education Program (NCEP) Expert Panel on Detection, Evaluation, And Treatment of High Blood Cholesterol In Adults (Adult Treatment Panel III). JAMA, 285: 2486-97.

Wood, P.D., Stern, M.P., Silvers, A., Reaven, G.M., von der Groeben, J. 1972. Prevalence of plasma lipoprotein abnormalities in a freeliving population of the Central Valley, California. Circulation, 45: 114-126. 